\title{
Peripheral artery disease: potential role of ACE-inhibitor therapy
}

\author{
Giuseppe Coppola \\ Giuseppe Romano \\ Egle Corrado \\ Rosa Maria Grisanti \\ Salvatore Novo \\ Department of Internal Medicine, \\ Cardiovascular and Nephro-Urological \\ Diseases, Chair of Cardiovascular \\ Disease, University of Palermo, \\ Palermo, Italy
}

\begin{abstract}
Subjects with peripheral arterial disease (PAD) of the lower limbs are at high risk for cardiovascular and cerebrovascular events and the prevalence of coronary artery disease in such patients is elevated. Recent studies have shown that regular use of cardiovascular medications, such as therapeutic and preventive agents for PAD patients, seems to be promising in reducing long-term mortality and morbidity. The angiotensin-converting-enzyme (ACE) system plays an important role in the pathogenesis and progression of atherosclerosis, and ACE-inhibitors (ACE-I) seem to have vasculoprotective and antiproliferative effects as well as a direct antiatherogenic effect. ACE-I also promote the degradation of bradykinin and the release of nitric oxide, a potent vasodilator; further, thay have shown important implications for vascular oxidative stress. Other studies have suggested that ACE-I may also improve endothelial dysfunction. ACE-I are useful for reducing the risk of cardiovascular events in clinical and subclinical PAD. Particularly, one agent of the class (ie, ramipril) has shown in many studies to able to significantly reduce cardiovascular morbidity and mortality in patients with PAD.
\end{abstract}

Keywords: atherosclerosis, peripheral arterial disease, endothelial dysfunction, ACE-inhibitors

\section{Introduction}

Peripheral arterial disease (PAD) of the lower limbs is the third most important site of atherosclerotic disease alongside coronary heart disease (CHD) and cerebrovascular disease (CVD) (Novo 1995). This clinical condition has often been neglected in the past but, in recent years, PAD has received growing attention as an important cause of disability and of cardiovascular morbidity and mortality (Novo and Coppola 2002; Novo 1995). Subjects with PAD represent a category of patients at a very high cardiovascular risk of fatal and non-fatal cerebrovascular and cardiovascular events; therefore, they need to be treated not only for local problems derived from arteriopathy (intermittent claudication, rest pain and/or ulcers) but, above all, for preventing vascular events (Clement et al 2000; Gibbons et al 2003; Bhatt et al 2006; Antman et al 2004). Simple non-invasive tests such as measurement of Ankle/Brachial pressure Index (ABI), the so-called Index of Winsor, and ankle and toe Doppler pressures represent easy and useful methodologies in clinical practice; in fact, such tests can be performed in only a few minutes and can provide sufficient information to confirm the diagnosis of PAD and to document the severity of limb ischemia (Dormandy and Rutherford 2000; Milio et al 2004). The echographic examination of carotid and peripheral atherosclerotic lesions may be useful in patients with PAD for identifying subjects at higher risk for cerebrovascular and cardiovascular events, and their early identification may favor more aggressive approaches of pharmacological treatment in order to avoid future events (Romano et al 2006). Recently, a number of studies have suggested that ramipril, an angiotensin-converting enzyme inhibitor (ACE-I), and statins, together with antiplatelet drugs, may reduce cardiovascular morbidity 
and mortality in PAD (Novo and Evola 2003; Coppola and Novo 2007).

ACE-I were developed as therapeutic agents for essential arterial hypertension. Since the initial application of these drugs, several additional clinical indications have been identified and approved (Brown and Vaughan 1998), such as reduction in mortality and hospitalizations for heart failure in patients with moderate left ventricular dysfunction, with and without signs or symptoms of congestive heart failure; benefits in patients with ischemic and non-ischemic cardiomyopathies and with or without recent myocardial infarction (SOLVD Investigators 1991; Pfeffer et al 1992); and reductions in left ventricular remodeling (Pfeffer et al 1988; Sharpe et al 1991). Recently, the role of the rennin-angiotensin-aldosterone system (RAAS) has been described in the pathogenesis and progression of atherosclerosis (Lonn et al 1994). Peripheral artery disease (PAD) is related to atherosclerotic stenosis and inappropriate dilatation or abnormal constriction of arteries and microcirculation (Meredith et al 1993; Hasdai et al 1997). Endothelial dysfunction represents one of the mechanisms involved in the disturbance of artery vasomotion. The central role of endothelium in vascular tone regulation is due to its ability to release both vasodilating and vasoconstricting substances. In animal models, ACE-I can retard the development of atherosclerosis, and these antiatherogenic properties can be related to the inhibition of angiotensin-II (Ang II) formation and to the inhibition of bradykinin degradation, which promotes vasodilatation by stimulating the production of arachidonic acid metabolites and nitric oxide (NO) in vascular endothelium. In summary, the ACE system regulates the balance between the vasodilatory properties of bradykinin and the vasoconstrictive properties of Ang II. ACE-I alter this balance by decreasing the formation of Ang II and the degradation of bradykinin (Figure 1): the bradykinin is potentiated and NO is released to a greater extent, resulting in decreased migration and proliferation of vascular smooth muscle cells, decreased accumulation and activation of inflammatory cells, decreased oxidative stress, and improved endothelial function.

\section{Vasculoprotective effects of ACE-inhibitors}

The vascular protective effects of ACE-I may be summarized as follows (Table 1).

\section{Antiproliferative effects}

ACE-I exhibit antiproliferative effects (reduction of vascular and cardiac hypertrophy and extracellular matrix proliferation) and reduce ventricular remodeling after myocardial infarction (Paul and Ganten 1992; Schiffrin and Deng 1995). In the hypertrophied heart ACE-I reduce cardiac hypertrophy and improve diastolic function. ACE-I also prevent apoptosis of cardiac myocytes in pressureoverloaded hearts.

In contrast to these antiproliferative and antimigratory effects, an enhancement of endothelial cell migration has

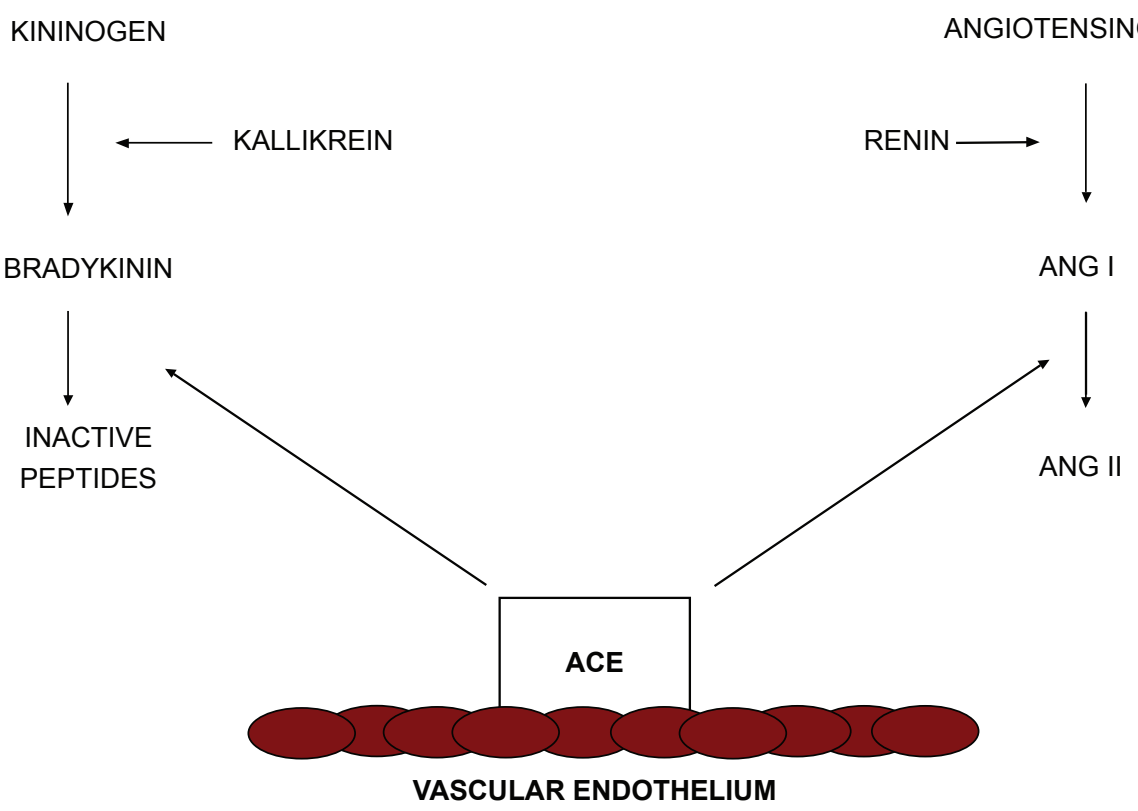

Figure I Renin-angiotensin system and kallikrein-kinin system. Angiotensin-converting enzymes regulate the balance between angiotensin-II (Ang II) and bradykinin. Adapted from Brown and Vaughan (1998). 
Table I Vasculoprotective effects of angiotensin-converting enzyme inhibitors (adapted from Lonn et al 1994)

\begin{tabular}{l} 
Vasculoprotective effects \\
\hline Direct antiatherogenic effect* \\
Antiproliferative and antimigratory effects on smooth muscle cells, \\
neutrophils and mononuclear cells \\
Improvement and/or restoration of endothelial function \\
Protection from plaque rupture* \\
Antiplatelet effects \\
Enhancement of endogenous fibrinolysis* \\
Antihypertensive effects \\
Improvement in arterial compliance and tone
\end{tabular}

*Not demonstrated conclusively in humans.

been demonstrated with ACE-I with reduced Ang II that may contribute to improved endothelial function and might therefore exert an antiatherosclerotic action.

\section{Effects on the fibrinolytic balance}

ACE-I also modulate the vascular fibrinolytic balance by decreasing Ang II, a potent activator of plasminogen activator inhibitor type 1 (PAI-1) synthesis, and by increasing levels of bradykinin, a potent activator for tissue plasminogen (Vaughan 1997). ACE-I lower plasminogen activator inhibitor type 1 (PAI-1) concentrations. PAI-1 represent the most important physiological inhibitor of tissue-type plasminogen activator (TPA) in plasma and elevated levels have been implicated in the pathogenesis of thromboembolic disease (Ridker 1992). It has been demonstrated that human platelets have Ang II receptors. The action of ACE-I on platelets could be related to Ang II blockade. Platelet aggregation may also be suppressed through increased prostacyclin induced by elevated bradykinin levels.

\section{Direct "antiatherogenic" effect}

Vascular and cardioprotective properties of ACE-I largely result from their effects in inhibiting Ang II formation. Generation of Ang II is regulated by a circulating RAAS and a tissue angiotensin system, both of which are upregulated in cardiovascular diseases (Dzau and Re 1994). Increased expression of tissue ACE has been found in the vasculature of subjects with hypertension and in the microvasculature throughout an atherosclerotic plaque, suggesting that the accumulation of ACE in the plaque contributes to increased local production of Ang II (Gibbons 1995; Diet et al 1996). ACE inhibition modifies the structure of the arterial wall and of vulnerable plaque, effects that might lead plaques to be less vulnerable to rupture.
A direct "antiatherogenic" action of these drugs has been shown in several animal models of atherosclerosis related to cholesterol-mediated endothelial injury and in models of accelerated atherosclerosis after mechanical endothelial damage (balloon endothelial injury).

Chobanian et al (1990) studied the effects of captopril in the normotensive Watanabe Heritable Hyperlipidemic (WHHL) rabbit, an experimental model in which other blood-pressure-lowering drugs such as propranolol, nifedipine and verapamil failed to inhibit the development of atherosclerotic lesions. It appears, therefore, that in addition to a reduction in the anatomic extent of atherosclerotic lesions, captopril had potentially stabilizing effects on the atherosclerotic lesions, which may be associated with less propensity to rupture. Rolland et al (1993) demonstrated a reduction in the atherosclerotic lesion size, a decrease in the lipid-laden macrophages and less fragmentation of the arterial elastic tissue in the Pitman-Moore minipig treated with the ACE-I perindopril and receiving a high-fat diet. The atherosclerotic lesions that developed in perindopril-treated animals appeared less prone to rupture and had improved viscoelastic properties, favoring improved arterial flow. Although these findings suggest potential benefits for the use of ACE-I in ischemic cardiovascular diseases beyond their hemodynamic effects, we need to view them with caution. The atherosclerotic plaques generated in animal models receiving high-cholesterol or high-fat diets are likely to differ from those observed in human atherosclerosis, so that the clinical impact of agents able to stabilize atherosclerotic lesions remains unclear.

\section{Endothelial dysfunction}

\section{and atherosclerosis}

The endothelium is a common target of all risk factors, and fuctional impairment of the vascular endothelium in response to injury occurs long before the development of atherosclerotic changes in arteries. Impaired endothelium-dependent vasodilation is an adverse prognostic parameter in patients with risk factors such as hypertension, diabetes, smoking, hypercholesterolemia and atherosclerotic vascular disease.

In patients with coronary heart disease (CHD), endothelial dysfunction predicts long-term atherosclerotic disease progression and cardiovascular event rates (Schächinger et al 2000).

A number of processes characterize endothelial dysfunction, enhanced vasoconstrictor responses, adhesion of platelets and monocytes, and proliferation and migration of vascular smooth muscle cells. 
ACE-I have been shown to improve or restore endothelial function in different animal models (Pepine 1998; Enseleit et al 2003) and in humans. The Trial on Reversing Endothelial Dysfunction (TREND) showed convincing evidence for an effect of ACE inhibition on the endothelium-dependent vasodilation in subjects with coronary heart disease (Mancini et al 1996). After 6 months of quinapril treatment, the initial vasoconstrictor response to intracoronary infusion of acetylcholine was reduced and the vasodilator response was restored. Only the quinapril group showed significant net improvement in response to incremental concentrations of acetylcholine $(p=0.002)$.

Galatius et al (1999) investigated the effect of 12 weeks of treatment with the ACE-I fosinopril on peripheral microvascular function in a double-blind, placebo-controlled study of 12 patients treated with fosinopril and 10 patients treated with placebo. All had moderate congestive heart failure (CHF). Skeletal muscle vascular resistance and skin minimal vascular resistance were reduced in the fosinopril group ( $p<0.05$ for both) while no changes were reported in the placebo group. These findings suggest that long-term ACE-I treatment with fosinopril in patients with CHF improves the hemodynamic status as far as the peripheral microvascular level in both the relaxed and non-relaxed microcirculation of the lower legs. Such an effect appears to be mediated primarily by bradykinin accumulation.

Bradykinin is a peptide that stimulates the synthesis and/or activity of NO by binding to bradykinin receptors on the surface of endothelial cells. Accumulation of bradykinin via inhibition of ACE may increase NO synthesis or activity, resulting in improved vasodilative response. $\mathrm{NO}$ is synthesized in endothelial cells by a constitutively expressed enzyme, called endothelial NO synthase (eNOS ), or its inducible isoform, inducible NO synthase (iNOS) (Hermann and Luscher 2006). NO is released from endothelial cells mainly in response to shear stress produced by blood flow or pharmacological stimulants such as acetylcholine. After diffusion from endothelial to vascular smooth muscle cells, NO increases intracellular cyclic guanosine-monophosphate (cGMP) concentrations leading to relaxation of the smooth muscle cells. Bradykinin also stimulates the endothelium to release prostacyclin (PGI-2) that is derived from the metabolism of arachidonic acid (Figure 2). PGI-2 relaxes most blood vessels by activating adenylyl cyclase and increasing cyclic adenosine monophosphate (cAMP) levels. PGI-2 is considered to be the main prostanoid synthesized

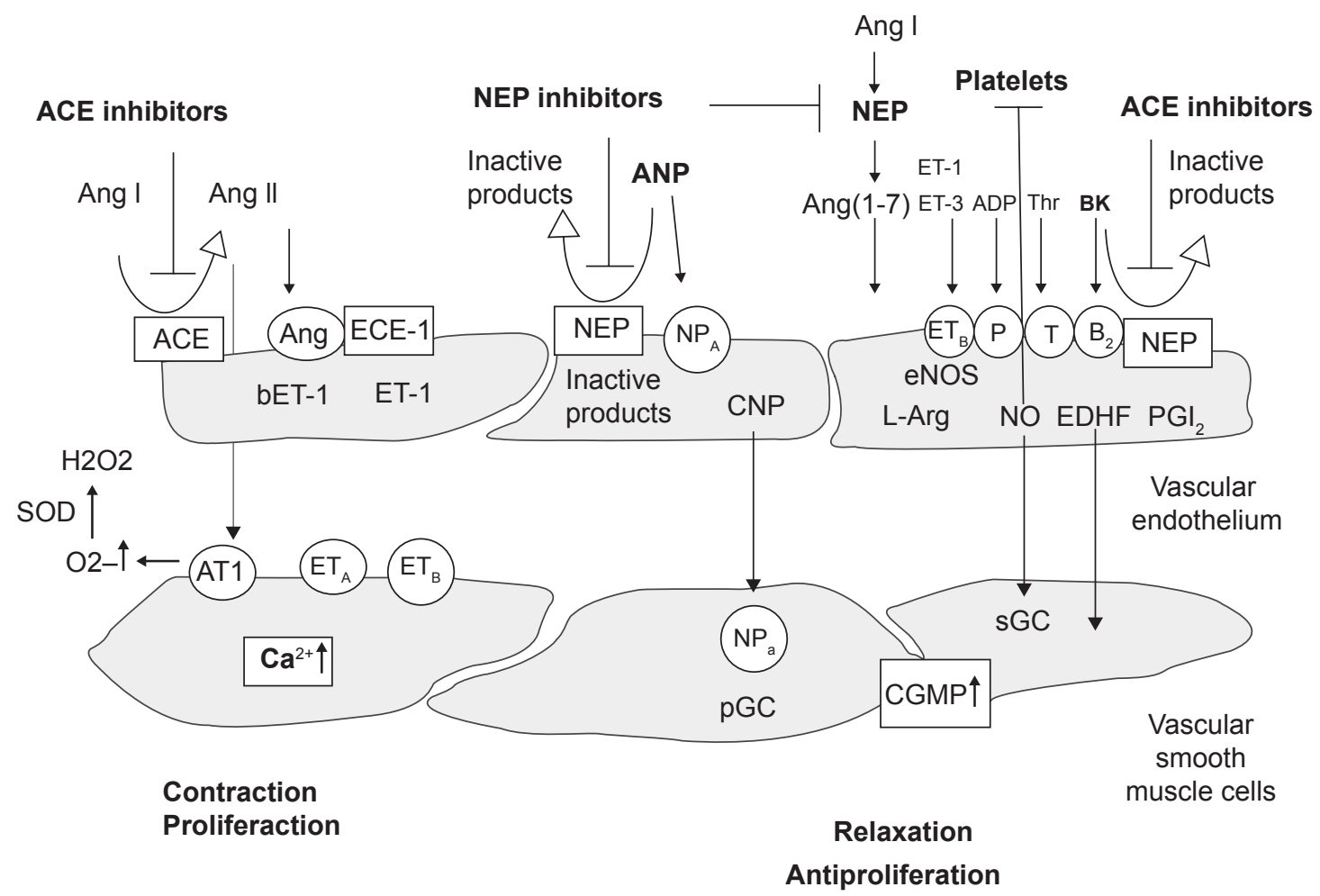

Figure 2 Endothelium-derived vasoactive substances.Various blood- and platelet-derived substances can activate specific receptors (open circles) on the endothelial membrane to release relaxing factors such as nitric oxide (NO), prostacyclin $\left(\mathrm{PGI}_{2}\right)$, and an endothelium-derived hyperpolarizing factor (EDHF). Furthermore, contracting factors are released, such as endothelin-I (ET-I) and angiotensin (Ang). Adapted from Enseleit et al (2003).

Abbreviations: ACE, angiotensin-converting enzyme; NEP, neutral endopeptidase;Thr, thrombin; bET-I, big-endothelin-I; Bk, bradykinin; TX, thromboxane; O2, superoxide. 
by vascular endothelium, while thromboxane A2 (TX-A2) is the main prostanoid produced by platelets.

Therefore, bradykinin may play a central role in preventing the development of proliferative atherosclerotic lesions in response to vascular injury (Cannon 1998).

\section{ACE-inhibitors and oxidative stress}

Increasing evidence suggests that ACE-I may have important implications for vascular oxidative stress (Münzel and Keaney 2001). Ang II stimulates the NADH/NAD(P)H (nicotinamide adenine dinucleotide/nicotinamide adenine dinucleotide phosphate) oxidases of endothelial and smooth muscle cells, resulting in increased generation of superoxide anions. The latter are able to degrade NO, with significant implications for atherosclerosis development and progression. Superoxide combines with NO in a diffusion limited reaction that is faster than the dismutation of superoxide by the superoxide dismutase (which produces $\mathrm{H}_{2} \mathrm{O}_{2}$ ). This reaction produces peroxynitrite, a compound that reduces $\mathrm{NO}$ bioactivity and also promotes lipid and protein oxidation in atherosclerotic lesions, which decreases the bioavailability of endothelium-derived NO (Cannon 1998). In addition, the ACE-I limits the stimulation of vascular NAD(P)H oxidase, thereby preventing the increased superoxide flux associated with activation of the RAAS. This should produce a number of important downstream effects that benefit the vasculature.

Because NO is known to inhibit the activity of NAD $(\mathrm{P}) \mathrm{H}$ oxidase, another predictable effect of ACE-I would be to reduce the ambient levels of superoxide in the vascular wall (Lee et al 2000). ACE inhibition should also inhibit lipid peroxidation through reduced formation of peroxynitrite. Since superoxide is the principal source of $\mathrm{H}_{2} \mathrm{O}_{2}$, an agent typically associated with lipid and protein oxidation and necrosis or apoptosis in a number of cell types, ACE-I should limit smooth muscle proliferation. The relationship between ACE action and vascular oxidative stress is summarized in Figure 3.

\section{Impact of ACE inhibition in PAD}

Only a few studies have directly evaluated the potential role of ACE-I in PAD. Peripheral arterial disease remains

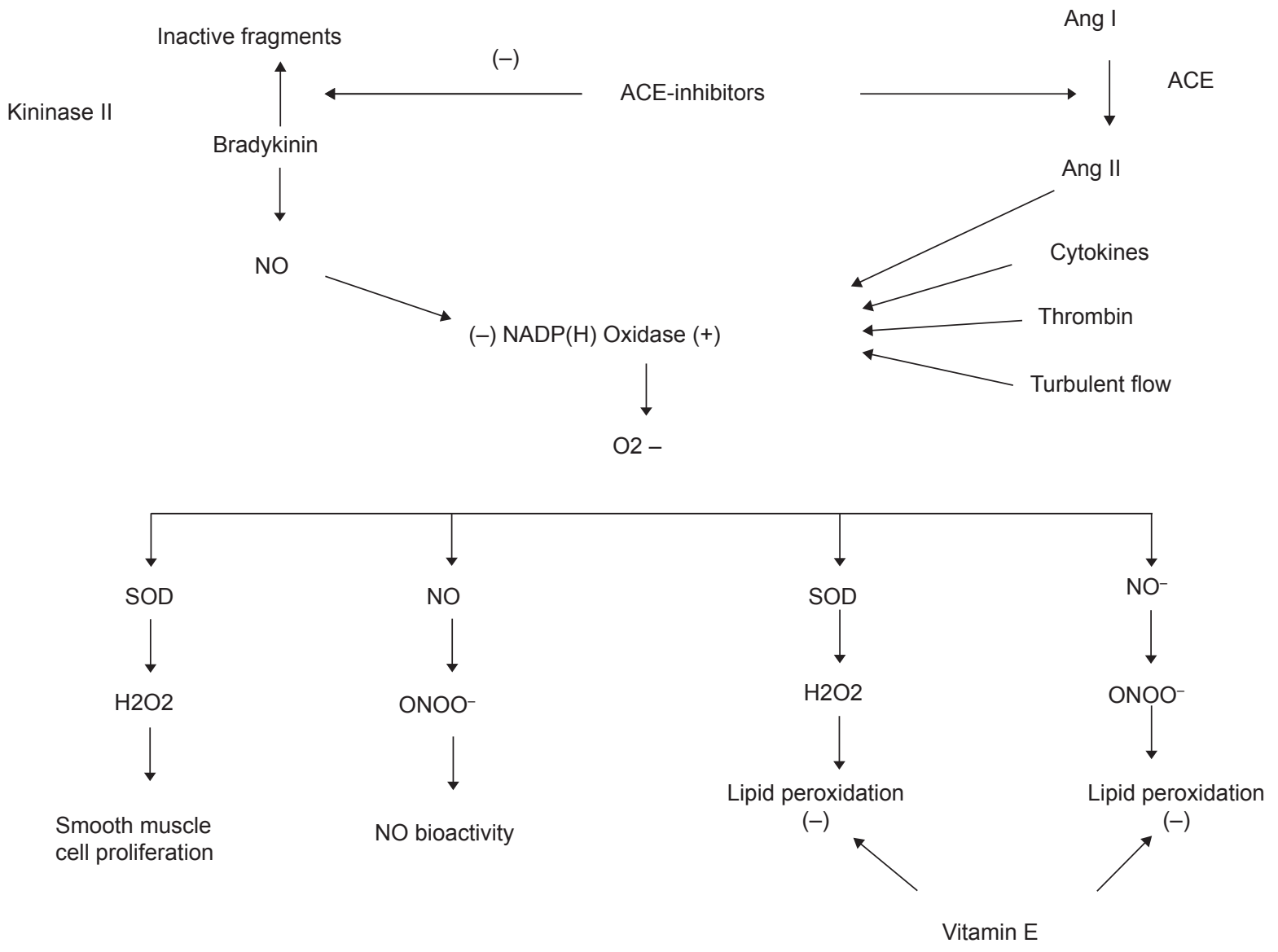

Figure 3 Scheme for relation between angiotensin-converting enzyme (ACE) action and vascular oxidative stress. ACE inhibitors both stimulate NO production and prevent formation of $\mathrm{O}_{2}^{-}, \mathrm{H}_{2} \mathrm{O}_{2}$, and $\mathrm{NO} / \mathrm{C}>2 \sim$ reaction product peroxynitrite (ONOO-), thereby abrogating a number of downstream effects. In contrast, radical scavenging antioxidant vitamin $\mathrm{E}$ is active only against certain components of oxidative stress, leaving other consequences of NAD(P)H oxidase activity untouched. Adapted from Münzel et al (200I). 
an underdiagnosed disease in primary care and patients are not treated as aggressively such as those with other ischemic forms of atherosclerotic disease (McDermott et al 2001; Belch et al 2003). Cardiovascular events are still the major cause of morbidity and mortality in patients with PAD; therefore the potential benefit of an aggressive anti-atherosclerotic therapy still remains ill defined.

Higashi et al (2000) conducted a large multicenter study in 296 patients with essential arterial hypertension, including 46 untreated subjects and 47 normotensive subjects. They compared calcium antagonists, beta-blockers, diuretics and ACE-I, evaluating the effects of these antihypertensive drugs on NO and prostaglandin release. After 24 weeks of treatment the reactive hyperemia was impaired in hypertensive patients compared with normotensive subjects $(\mathrm{p}<0.01)$. Reactive hyperemia was greater in hypertensive patients treated with ACE-I than in those treated with calcium antagonists $(\mathrm{p}<0.01)$, beta-blockers $(\mathrm{p}<0.01)$, diuretic agents $(\mathrm{p}<0.05)$ and untreated hypertensive patients $(\mathrm{p}<0.01)$. The increase in FBF (forearm blood flow) after administration of sublingual nitroglycerin (NTG) was similar in all groups. The maximal FBF response from reactive hyperemia was significantly greater in the ACE-I treated group than in the calcium antagonist, beta-blocker or diuretic agent groups or in untreated hypertensive subjects $(\mathrm{p}<0.05)$, but was similar among the last four groups. In this study all drugs were equally effective in reducing blood pressure, but only ACE-I improved endothelial dysfunction.

Ostergren et al (2004) evaluated the measurement of ABI as a predictor of cardiovascular events and the effect of ramipril treatment on prognosis in patients with symptomatic PAD in relation to those with different ABI but without symptomatic PAD. Clinical events in patients randomized to ramipril and placebo respectively for primary outcome (cardiovascular mortality, myocardial infarction, stroke) was $20.1 \%$ for ramipril and $25.8 \%$ for placebo, in clinical PAD, and $13.6 \%$ for ramipril and $17.0 \%$ for placebo, in non-symptomatic PAD ( $\mathrm{p}=0.53)$. In patients with clinical PAD, the incidence of myocardial infarction was $12.3 \%$ in the ramipril group and $16.1 \%$ in the placebo group, vs $10.0 \%$ in the ramipril group vs $12.3 \%$ for placebo in nonsymptomatic PAD ( $\mathrm{p}=0.56)$. In patients with clinical PAD, the incidence of stroke was $6.2 \%$ for ramipril and $8.3 \%$ for placebo, vs 3.1\% for ramipril and $4.7 \%$ for placebo in non-symptomatic PAD ( $\mathrm{p}=0.75)$. The cardiovascular mortality was $10.4 \%$ for ramipril and $13.6 \%$ for placebo, in clinical PAD, vs 5.5\% for ramipril and 7.2\% for placebo in non-symptomatic PAD ( $p=0.79)$. All cause death was $16.7 \%$ for ramipril and $19.4 \%$ for placebo, in clinical PAD, and 9.5\% for ramipril and $10.9 \%$ for placebo, in non-symptomatic PAD $(p=0.39)$. The incidence of revascularization was $25.2 \%$ for ramipril and $27.7 \%$ for placebo, in clinical PAD, and $15.5 \%$ for ramipril and $17.7 \%$ for placebo in no symptomatic $\operatorname{PAD}(\mathrm{p}=0.78)$. The incidence diabetic complications was $22.1 \%$ for ramipril and $26.7 \%$ for placebo, in clinical PAD, and $15.0 \%$ for ramipril and $17.0 \%$ for placebo in no symptomatic PAD ( $\mathrm{p}=0.82)$. The incidence of hospitalizations for CHF was 5.0\% for ramipril and 6.6\% for placebo, in clinical PAD, and $2.8 \%$ for ramipril and $3.1 \%$ for placebo in non-symptomatic PAD $(p=0.22)$. In particular the relative benefit was similar in patients subdivided by levels of ABI (RR of 0.83 with $\mathrm{ABI}>0.9$, RR of 0.75 with $\mathrm{ABI}$ of $0.6-0.9$ and $\mathrm{RR}$ of 0.79 in those with $\mathrm{ABI}<0.6$; $\mathrm{p}$ for heterogeneity was not significant). Although not reaching the statistical significance, ramipril reduced the risk of cardiovascular events in all groups of patients with clinical PAD and with subclinical PAD regardless of ABI.

Feringa et al (2006) sought to determine the effect of chronic treatment with cardiac medication, including statins, beta-blockers, aspirins, ACE-I, calcium channel blockers, diuretics, nitrates, coumarins, and digoxin on long-term mortality among patients with PAD (Feringa et al 2006). Statins, beta-blockers, aspirin and ACE-I are associated with a reduction in long-term mortality risk in patients with PAD that is independent of clinical risk factors. During a median follow-up of 8 years, 1067 patients (44\%) died. After adjustment for risk factors and propensity scores ACE-I (HR 0.80, 95\% CI 0.69-0.94) were significantly associated with a reduced risk of long-term mortality. The use of cardiac medications as therapeutic and preventive agents in patients with PAD seems to be promising in reducing long-term mortality and could be incorporated among other management strategies, including walking exercise and risk factor modification.

The HOPE Study has demonstrated that ramipril is beneficial in a broad range of patients without evidence of left ventricular systolic dysfunction or heart failure who are at high risk for cardiovascular events (HOPE 2000). Treatment with ramipril reduced the rates of death from cardiovascular causes $(6.1 \%$ compared with $8.1 \%$ in the placebo group; RR 0.74; p $<0.001)$, myocardial infarction (9.9\% vs $12.3 \%$; RR $0.80 ; \mathrm{p}<0.001)$, stroke $(3.4 \%$ vs $4.9 \%$; RR 0.68 ; $\mathrm{p}<0.001)$, death from any cause $(10.4 \%$ vs $12.2 \%$; RR 0.84 ; $\mathrm{p}=0.005)$, revascularization procedures $(16.0 \%$ vs $18.3 \%$; RR $0.85 ; \mathrm{p}=0.002)$, cardiac arrest $(0.8 \%$ vs $1.3 \%$; RR 0.63 ; $\mathrm{p}=0.03)$, heart failure (9.0\% vs $11.5 \%$; RR $0.77 ; \mathrm{p}<0.001)$, 
and complications related to diabetes $(6.4 \%$ vs $7.6 \%$; RR 0.84; $\mathrm{p}=0.03$ ).

The underlying rationale for the study was that the inhibition of ACE would prevent events related to ischemia and atherosclerosis; the study therefore included a broad range of patients with any atherosclerosis manifestation: coronary artery disease (CAD), history of CVD or peripheral vascular disease, or diabetes and one cardiovascular risk factor; ramipril was beneficial in all these subgroups. The benefits of ramipril were observed among patients who were already taking a number of effective treatments, such as aspirin, beta-blockers and lipid lowering agents, indicating that the inhibition of ACE offers an additional approach to the prevention of atherothrombotic complications. Only a small part of the benefit could be attributed to a reduction in blood pressure, since the majority of patients did not have hypertension at baseline (according to conventional definitions) and the mean reduction in blood pressure with treatment was extremely small. However, in this study there was a $22 \%$ risk reduction in patients randomized to ramipril compared with placebo, which was independent of lowering of blood pressure. Based on this finding, the US Federal Drug Administration has now approved ramipril for its cardioprotective benefits in patients at high risk, including those with PAD. Thus, considering this like a drug class effect, the ACE-I would be recommended in patients with PAD.

The Study to Evaluate Carotid Ultrasound Changes in Patients Treated with Ramipril and Vitamin E (SECURE), a HOPE substudy, was a prospective, double-blind, 332-patient factorial design trial that evaluated the effects of long-term treatment with the ACE-I ramipril and vitamin E on atherosclerosis progression in high-risk patients (Lonn et al 2001). The primary study analysis included 693 patients. There was an overall effect of ramipril, which reduced the annualized slope of the mean maximum IMT (intimal medial thickness) versus ramipril placebo $(p=0.033)$, and there was a strong trend for benefit in the ramipril $10 \mathrm{mg}$ /day group versus ramipril placebo $(p=0.028)$. There was a reduced atherosclerosis progression rate for ramipril overall versus ramipril placebo $(\mathrm{p}=0.019)$ and for the ramipril $10 \mathrm{mg} /$ day group versus ramipril placebo $(p=0.015)$. In the analysis of the 637 patients who completed carotid ultrasonography examinations, ramipril had a highly significant effect on the single maximum IMT slope $(p=0.003$ for the overall effect of ramipril versus placebo and $p=0.008$ for ramipril $10 \mathrm{mg}$ /day versus placebo). This trial shows that long-term therapy with ramipril reduced atherosclerosis progression rates. This effect was noted in high-risk patients, the majority of whom were already on effective therapy. Although the absolute differences in atherosclerosis progression rates between ramipril- and placebo-treated patients are small, the relative reduction in mean maximum IMT was 37\% for ramipril $10 \mathrm{mg} /$ day versus placebo, which is similar to the $32 \%$ reduction in the risk of stroke in HOPE.

Ahimastos et al (2006) demonstrated that ramipril improves walking ability in patients with PAD. They examined the effect of 6-month ramipril therapy (10 mg of ramipril once daily) on walking distance and claudication, in a defined group of patients without diabetes and with claudication due to infrainguinal PAD, by using a double-bind, randomized, placebo-controlled design. This study was conducted on 152 patients with PAD, of whom 40 were recruited and completed the trial. All patients had an ABI less than 0.9 at rest in at least $1 \mathrm{leg}$, had a history of intermittent claudication, which was stable for 6 months and had evidence of superficial femoral artery stenosis or occlusion on duplex ultrasonography. All patients performed a treadmill exercise test to evaluate pain-free walking time and maximum walking time. The ramipril and placebo groups were similar in age, other cardiovascular risk factors, medication use and PAD severity, as evidenced by clinical symptoms (walking times), WIQ (Walking Impairment Questionnaire) scores and resting $\mathrm{ABI}$. The study showed that ramipril increased ABI both at rest $(p<0.001)$ and after exercise $(p<0.001)$. At rest, this increase was due to reduction in brachial systolic blood pressure with ramipril treatment rather than an increase in limiting leg pressure. After exercise, both a reduction in brachial pressure $(\mathrm{p}<0.001)$ and an increase in limiting leg pressure $(p<0.001)$ contributed to the increase in ABI. After adjuststment for the baseline pain-free walking time, mean pain-free walking time after ramipril treatment was 227 seconds (95\% CI, 175-278 seconds; p < 0.001) longer than that after placebo treatment. Similarly, maximum walking time improved by 451 seconds in the ramipril group (CI, 367 seconds to 536 seconds; $\mathrm{p}<0.001$ ) but did not change in the placebo group. The magnitude of this effect is greater than that reported for convenctional medical therapies. Moreover, ramipril significantly increased volume flow in the common femoral artery proximal to the site of stenosis in both the limiting leg $(p<0.008)$ and the non-limiting leg $(\mathrm{p}<0.035)$. In this trial ramipril improved WIQ median distance score from 5\% (range, 1\%-39\%) to $21 \%$ (range, $12 \%-58 \%$; $<<0.001$ ), speed score from $3 \%$ (range, $3 \%$ to $39 \%$ ) to $18 \%$ (range, $8 \%$ to $50 \%$; p < 0.001 ), and stair-climbing score from $17 \%$ (range, 4\%-80\%) to $67 \%$ (range, $38 \%-88 \%$; $<0.001$ ). In conclusion, ramipril therapy for 24 weeks increased both pain-free walking time 
and maximum walking time by a clinically and statistically significantly greater magnitude than current therapies.

Another study by Ahimastos et al (2008) supports the hypothesis that the beneficial effects of ramipril on pain-free walking time observed in a PAD population are, at least partly, a consequence of reduced arterial stiffness. In this double-bind study, 40 patients with $P A D$ were randomized to ramipril, $10 \mathrm{mg}$ once daily or placebo for 24 weeks. In this study pain-free walking time was recorded during a standard treadmill test, indices of arterial stiffness were assessed globally by systemic arterial compliance and augmentation index and regionally via central pulse wave velocity. They found that ramipril increased maximum walking time by $243 \%$ and improved arterial stiffness parameters by between $17 \%$ and $64 \%$ (all $p<0.001$ compared with placebo). There were moderately strong correlations between the pre-/post-intervention change in maximum walking time and in indices of arterial stiffness (systemic arterial compliance, $r=0.65, p<0.001$; central pulse wave velocity, $r=-0.57$, $\mathrm{p}<0.001$; augmentation index, $\mathrm{r}=-0.79, \mathrm{p}<0.001$; time to pressure augmentation, $\mathrm{r}=0.52, \mathrm{p}<0.001$ ).

\section{Conclusions}

The imbalance between NO and Ang II activities associated with endothelial dysfunction and risk factors for CAD causes oxidative stress, which results from excessive production of oxygen-free radicals which counteracts the effects of NO, stimulates expression of adhesion molecules and promotes adhesion of leukocytes to the endothelium. These actions cause an acute inflammatory response, proliferation of smooth muscle cells and production of extracellular matrix, all of which contribute to the pathogenesis of cardiovascular disease (Griendling and Alexander 1997). The antiatherogenic ACE-I properties may be related both to the inhibition of tissue and circulating Ang II formation and to bradykinin potentiation, resulting in decreased proliferation and migration of smooth muscle cells, decreased accumulation and activation of inflammatory cells, decreased oxidative stress, and increased endothelial NO formation, leading to improved endothelial function. The remarkable improvement in the long-term prognosis of atherosclerotic patients with increased cardiovascular risk might be the clinical result of the contribution made by ACE inhibition in the vascular wall. On the basis of this finding, the latest ACC/AHA 2005 practice Guidelines for the Management of Patients With Peripheral Arterial Disease have suggested that the use of ACE-I is reasonable for symptomatic patients with lower extremity PAD in order to reduce the risk of adverse cardiovascular events (class IIa, Level of Evidence: B) and that ACE-I may be considered for patients with asymptomatic lower extremity PAD to reduce the risk of adverse cardiovascular events (class IIb, Level of Evidence: C) (Hirsch et al 2006). Yet, availability of newer neurohormonal antagonists such as angiotensin receptor blockers, beta-blockers and aldosterone antagonists, lack of industry sponsorship for ACE-I, and aggressive promotion of statin have further slighted the role of this useful, safe and affective drug not only in the prevention of atherosclerosis vascular disease but also in improving walking ability in patients with PAD as consequence of reduced arterial stiffness. There is currently no evidence for the efficacy of ACE-I in patients with asymptomatic PAD, and, thus, the use of ACE-I medications to lower cardiovascular ischemic event rates in this population must be extrapolated from the data on symptomatic patients. The recent guidelines of the Trans-Atlantic Inter Society Consensus Document on Management of PAD (TASC II) have recommended the control of hypertension in PAD patients, maintaining blood pressure values $<140 / 90 \mathrm{mmHg}$ or $<130 / 80 \mathrm{mmHg}$ if they have diabetes or renal insufficiency (Grade A) (Norgren et al 2007). The ACE-I should be considered as initial blood-pressure lowering drugs in PAD to reduce the risk of cardiovascular events (Grade B). Another recommendation regards the management of CAD in PAD patients. These patients with clinical evidence of CAD (angina, ischemic congestive heart failure) should be evaluated and managed according to current guidelines (Grade C). Patients with PAD considered for vascular surgery may undergo further risk stratification and those found to be at very high risk managed according to current guidelines for coronary revascularization (Grade C).

\section{Disclosures}

The authors have no conflicts of interest to disclose.

\section{References}

Ahimastos A, Lawler A, Reid C, et al. 2006. Ramipril markedly improves walking ability in patients with peripheral arterial disease. Ann Intern Med, 144:660-4.

Ahimastos AA, Dart AM, Lawler A, et al. 2008. Reduced arterial stiffness may contribute to angiotensin-converting enzyme inhibitor induced improvements in walking time in peripheral arterial disease patient. J Hypertens, 26:1037-42.

Antman EM, Anbe DT, Armstrong PW, et al. 2004. ACC/AHA guidelines for the management of patients with ST-elevation myocardial infarction: a report of the American College of Cardiology/American Heart Association Task Force on Practice Guidelines (Committee to Revise the 1999 Guidelines for the Management of Patients with Acute Myocardial Infarction). Circulation, 110:e82-e292.

Belch JJ, Topol EJ, Agnelli G, et al. 2003. Prevention of Atherothrombotic Disease Network. Critical issues in peripheral arterial disease detection and management: a call to action. Arch Intern Med, 163:884-92.

Bhatt D, Steg P, Ohman E, et al. 2006. International prevalence, recognition, and treatment of cardiovascular risk factors in outpatients with atherothrombosis. JAMA, 295:180-9.

Brown NJ, Vaughan DE. 1998. Angiotensin-converting enzyme inhibitors. Circulation, 97:1411-20. 
Cannon RO. 1998. Potential mechanisms for the effect of angiotensin-converting enzyme inhibitors on endothelial dysfunction: the role of nitric oxide. Am J Cardiol, 82:8S-10S.

Chobanian AV, Haudenschild CC, Nickerson C, et al. 1990. Antiatherogenic effect of captopril in the Watanabe heritable hyperlipidemic rabbit. Hypertension, 15:327-31.

Clement DL, Boccalon H, Dormandy J, et al. 2000. A clinical approach to the management of the patient with coronary $(\mathrm{Co})$ and/or carotid $(\mathrm{Ca})$ artery disease who presents with leg ischaemia (Lis). Int Angiol, 19:97-125.

Coppola G, Novo S. 2007. Statins and peripheral arterial disease: effects on claudication, disease progression, and prevention of cardiovascular events. Arch Med Res, 38:479-e488.

Diet F, Pratt RE, Berry GJ, et al. 1996. Increased accumulation of tissue ACE in human atherosclerotic coronary artery disease. Circulation, 94:2756-67.

Dormandy JA, Rutherford RB. 2000. Management of peripheral arterial disease (PAD). TransAtlantic Inter- Society Consensus (TASC). $J$ Vasc Surg, 31:S1e-S296.

Dzau VJ, Re R. 1994. Tissue angiotensin system in cardiovascular medicine: a paradigm shift? Circulation, 89:493-8.

Enseleit F, Lüscher TF, Ruschitzka F. 2003. Angiotensin-converting enzyme inhibition and endothelial dysfunction: focus on ramipril. Eur Heart $J$ Suppl, 5(Suppl A):A31-A36.

Feringa HH, van Waning VH, Bax JJ, et al. 2006. Cardioprotective medication is associated with improved survival in patients with peripheral arterial disease. J Am Coll Cardiol, 47:1182-7.

Galatius S, Wroblewski H, Sorensen V, et al. 1999. Reversal of peripheral microvascular dysfunction during long-term treatment with the angiotensin-converting enzyme inhibitor fosinopril in congestive heart failure. J Card Fail, 5:17-24.

Gibbons GH. 1995. Mechanisms of vascular remodeling in hypertension: role of autocrine-paracrine vasoactive factors. Curr Opin Nephrol Hypertens, 4:189-96.

Gibbons RJ, Abrams J, Chatterjee K, et al. 2003. ACC/AHA 2002 guideline update for the management of patients with chronic stable angina - summary article: a report of the American College of Cardiology/American Heart Association Task Force on practice guidelines (Committee on the Management of Patients With Chronic Stable Angina). J Am Coll Cardiol, 41:159-68.

Griendling KK, Alexander RW. 1997. Oxidative stress and cardiovascular disease. Circulation, 96:3264-5.

Hasdai D, Gibbons RJ, Holmes DR Jr, et al. 1997. Coronary endothelial dysfunction in humans is associated with myocardial perfusion defects. Circulation, 96:3390-5.

Hermann M, Luscher TF. 2006. Endothelial dysfunction: the crux of the cardiovascular disease continuum. Medicographia, 28:318-25.

Higashi Y, Sasaki S, Nakagawa K, et al. 2000. A comparison of angiotensin-converting enzyme inhibitors, calcium antagonists, betablockers and diuretic agents on reactive hyperemia in patients with essential hypertension: a multicenter study. J Am Coll Cardiol, 35:284-91.

Hirsch AT, Haskal ZJ, Hertzer NR, et al. 2006. ACC/AHA 2005 practice Guidelines for the Management of Patients With Peripheral Arterial Disease (Lower Extremity, Renal, Mesenteric, and Abdominal Aortic). Circulation, 113:1474-547.

Lee C, Miura K, Liu X, et al. 2000. Biphasic regulation of leukocyte superoxide generation by nitric oxide and peroxynitrite. $J$ Biol Chem, 275:38965-72.

Lonn E, Yusuf S, Dzavik V, et al. 2001. Effects of ramipril and vitamin E on atherosclerosis: the Study to Evaluate Carotid Ultrasound Changes in Patients Treated With Ramipril and Vitamin E (SECURE). Circulation, 103:919-25.

Lonn EM, Yusuf S, Jha P, et al. 1994. Emerging role of angiotensinconverting enzyme inhibitors in cardiac and vascular protection. Circulation, 90:2056-69.

Mancini GB, Henry GC, Macaya C, et al. 1996. Angiotensin-converting enzyme inhibition with quinapril improves endothelial vasomotor dysfunction in patients with coronary artery disease. The TREND (Trial on Reversing ENdothelial Dysfunction) Study. Circulation, 94:258-65.
McDermott MM, Kerwin DR, Liu K, et al. 2001. Prevalence and significante of unrecognized lower extremity peripheral arterial disease in general medicine practice. $J$ Gen Intern Med, 16:384-90.

Meredith IT, Yeung AC, Weidinger FF, et al. 1993. Role of impaired endothelium-dependent vasodilation in ischemic manifestations of coronary artery disease. Circulation, 87(Suppl V):V56-V66.

Milio G, Coppola G, Genova. 2004. Ischemia cronica critica e vascolopatia ischemica polidistrettuale. Atti del Congresso XXV Congresso Sezione Regionale Siciliana, Sciacca, 30 Sept - 2 Oct 2004.

Münzel T, Keaney JF. 2001. Are ACE inhibitors a "magic bullet" against oxidative stress? Circulation, 104:1571-4.

Norgren L, Hiatt WR, Dormandy JA, et al. 2007. Management of peripheral arterial disease (PAD). TransAtlantic Inter-Society Consensus (TASC II). Int Angiol, 26:81-157.

Novo S, Coppola G. 2002. The Cardiovascular Risk Burden of Peripheral Arterial Disease (PAD). Invited Reports from the 17th International Congress on Thrombosis, Monduzzi Editore S.p.A. e MEDIMOND Inc; CX26R9044:231e8.

Novo S, Evola G. 2003. Role of statins and angiotensin converting enzyme inhibitors (ACE-I) in the therapy peripheral arterial disease. Int Angiol, 22(Suppl 1e2):45-e48.

Novo S. 1995. The patient with intermittent claudication. Everyday problem in clinical cardiology. Amsterdam: Excerpta Medica.

Ostergren J, Sleightb P, Dagenaisc G, et al. 2004. for the HOPE study investigators. Impact of ramipril in patients with evidence of clinical or subclinical peripheral arterial disease. Eur Heart J, 25:17-24.

Paul M, Ganten D. 1992. The molecular basis of cardiovascular hypertrophy: the role of the renin-angiotensin system. J Cardiovasc Pharmacol, 19:S51-8.

Pepine CJ. 1998. The effects of angiotensin-converting enzyme inhibition on endothelial dysfunction: potential role in myocardial ischemia. $\mathrm{Am}$ J Cardiol, 82:23S-7S.

Pfeffer MA, Braunwald E, Moyé LA, et al. 1992. on behalf of the SAVE Investigators. Effect of captopril on mortality and morbidity in patients with left ventricular dysfunction after myocardial infarction: results of the Survival and Ventricular Enlargement Trial. $N$ Engl J Med, 327:669-77.

Pfeffer MA, Lamas GA, Vaughan DE, et al. 1988. Effect of captopril on progressive ventricular dilation after anterior myocardial infarction. N Engl J Med, 319:80-6.

Ridker PM. 1992. An epidemiologic assessment of thrombotic risk factors for cardiovascular disease. Curr Opin Lipidol, 3:285-290.

Rolland PH, Charpiot P, Friggi A, et al. 1993. Effects of angiotensinconverting enzyme inhibition with perindolol on hemodynamics, arterial structure, and wall rheology in the hindquarters of atherosclerotic minipigs. Am J Cardiol, 71:22E-27E.

Romano G, Corrado E, Muratori I, et al. 2006. Carotid and peripheral atherosclerosis in patients who underwent primary percutaneous coronary intervention and outcome associated with multifocal atherosclerosis. Int Angiol, 25:389-94.

Schächinger V, Britten MB, Zeiher AM. 2000. Prognostic impact of coronary vasodilator dysfunction on adverse long-term out come of coronary heart disease. Circulation, 101:1899-906.

Schiffrin E, Deng L. 1995. Comparison of effects of angiotensin I-converting enzyme inhibition and b-blocade for two years on function of small arteries from hypertensive patients. Hypertension, 25:699-703.

Sharpe N, Smith H, Murphy J, et al. 1991. Early prevention of left ventricular dysfunction after myocardial infarction with angiotensin-convertingenzyme inhibition. Lancet, 337:872-6.

The Heart Outcomes Prevention Evaluation (HOPE) Study Investigators. 2000. Effects of an angio tensin converting-enzyme inhibitor, ramipril, on cardiovascular events in high-risk patients. $N$ Engl J Med, 342:145-53.

The SOLVD Investigators. 1991. Effect of enalapril on survival in patients with reduced left ventricular ejection fractions and congestive heart failure. N Engl J Med, 325:293-302.

Vaughan D. 1997. The renin-angiotensin system and fibrinolysis. Am J Cardiol, 79(Suppl 5):12-6. 
